\title{
Cervical Spine Fracture after a Bone Cracking Traditional (Tui Na) Massage
}

\section{Dear Editor,}

“Tui Na” (推拿) or traditional chinese medicine (TCM) massage is an alternative medicine therapy practised in many Chinese communities for the management of musculoskeletal ailments. During the massage, TCM practitioners utilise their fingers, hands, elbows, knees and/or feet to exert mechanical force on the patient's body to stimulate acupuncture points. This process is believed to restore the flow of the vital energy 'qi' throughout the meridians of the body, thereby enabling healing. ${ }^{1}$ While non-invasive and relatively harmless, the force applied during Tui $\mathrm{Na}$ has been known to cause complications of epidural haematoma, ${ }^{2}$ cerebral vascular accident ${ }^{3}$ and prolapsed intervertebral discs. ${ }^{4}$ In rare cases, vertebral fractures have also been reported. Here we report a local case of cervical vertebral fracture in an elderly lady after Tui Na massage, which was subsequently treated by surgical intervention involving anterior cervical corpectomy and posterior instrumentation.

A 73-year-old woman presented to our accident and emergency department with a 3-day history of neck pain and stiffness following a neck "cracking" Tui Na. This was preceded by a history of occasional mild axial neck pain for 2 years without any radicular nor myelopathic symptoms. There were no previously diagnosed cervical disorders in this patient. The massage was conducted by a TCM practitioner near her temple using only his hands. During the massage, the patient underwent forceful manipulation and compressive pressure applied on her neck in multiple directions. She did not note a particular manoeuvre that triggered the pain. After the massage, she experienced progressively worsening severe neck pain and stiffness and decided to seek medical treatment. She did not report any radiation of pain, limb weakness or numbness, urinary retention or bowel incontinence. There was no other reported fall or further trauma to the neck. The patient was not known to have any other medical conditions and was not on any long-term medications. She was also not taking any traditional medications nor supplements. Functionally, she was independent in all her activities of daily living and was community ambulant without aid prior to her TCM treatment
On clinical examination, her neck was held in forward flexed posture with lower cervical spine midline tenderness and paravertebral spasms. Range of motion of her neck was limited in all directions, particularly in flexion and extension. Muscle tones, reflexes, power and sensation were normal throughout all 4 limbs. Preserved anal sphincter tone and perianal sensation were confirmed by digital rectal examination.

Plain radiographs of the neck showed C6 anterior body compression fracture with kyphosis and widening of the C5-C6 interspinous distance (Fig. 1A, B). A C6 spinous process fracture and cortical irregularity of the C7 superior articulating facet was also noted.

A computer tomography (CT) scan confirmed a C6 vertebral anteroinferior corner fracture and loss of vertebral body height suggestive of a flexion teardrop fracture without posterior vertebral body retropulsion (Fig. 1C). Magnetic resonance imaging (MRI) revealed C5-C6 interspinous ligament oedema and widening, suggesting interspinous ligament injury and posterior column involvement (Fig. 1D, E, F). Short tau inversion recovery (STIR) images of the MRI revealed high signal intensity in the interspinous ligament between $\mathrm{C} 5$ and C6, where there was a transverse fracture in the body of the C6 spinous process, and high signal intensity in the $\mathrm{C} 6$ vertebral body (Fig. 1D). Pre-existing mild retrolisthesis was seen on $\mathrm{C} 3-\mathrm{C} 4, \mathrm{C} 4-\mathrm{C} 5$ and $\mathrm{C} 5-\mathrm{C} 6$. Degenerative disco-vertebral changes were present most notably on $\mathrm{C} 4-\mathrm{C} 5$ and $\mathrm{C} 5-\mathrm{C} 6$ with spinal stenosis and cord oedema.

Based on the clinical assessment and radiological investigation, she was diagnosed with an unstable $\mathrm{C} 6$ flexion teardrop fracture; $\mathrm{AO}$ classification: ${ }^{5} \mathrm{C} 5-\mathrm{C} 6$ of $\mathrm{B} 2$, due to the fracture configuration demonstrating a combined failure of both the posterior tension system and the anterior compressive column. The Sub-axial Cervical Spine Injury Classification (SLIC) score $^{6}$ is 3; morphology 1, disco-ligamentous complex (DLC) 2 ; neurological status 0 . She subsequently underwent cervical corpectomy and reconstruction of the C6 vertebral body and $\mathrm{C} 5-\mathrm{C} 7$ posterior instrumentation (Fig. 1G, H). She was immobilised in a cervical collar 
post-operatively. Direct visualisation of the cervical structures intraoperatively confirmed final diagnosis of flexion teardrop fracture and posterior tension band disruption.

A bone mineral density (BMD) test was performed and reported $\mathrm{T}$ scores of -2.4 to -3.0 , revealing severe osteoporosis. She was started on calcium and vitamin D supplements and subcutaneous teriparatide injections. The patient remained well and stable and was discharged from our institution on day 7 post-operatively, to a stepdown care facility for continued rehabilitation.

Injuries of the cervical spine and neurological system after external manipulation such as Tui $\mathrm{Na}$, massage or chiropractic have been reported, indicating the potential for injuries in these traditional healing methods. ${ }^{2-4,7-10}$ This is the first locally reported cervical fracture in an osteoporotic patient due to external spinal manipulation. In our literature review, we did not find reports of cervical fracture in osteoporotic patients from external spinal manipulation. There were, however, multiple reports of cervical spine fractures in osteoporotic patients after other forms of minor trauma and even spontaneous fractures with no apparent trauma. ${ }^{9}$

The mid-sagittal view of the STIR image (Fig. 1D) in the pre-operative MRI showed high signal intensity in the interspinous ligament between $\mathrm{C} 5$ and C6, where there was a transverse fracture in the body of the C6 spinous process, and high signal intensity in the C6 body. This corresponded to the upright view X-rays (Fig. 1A, B), showing the distraction between the C5 and C6 spinous processes and the wedge compression fracture of C6. The fracture configuration demonstrated a combined failure of both the posterior tension system and the anterior compressive column, hence a need for both anterior and posterior stabilisation. For SLIC scoring, we feel that the severity of the injury might
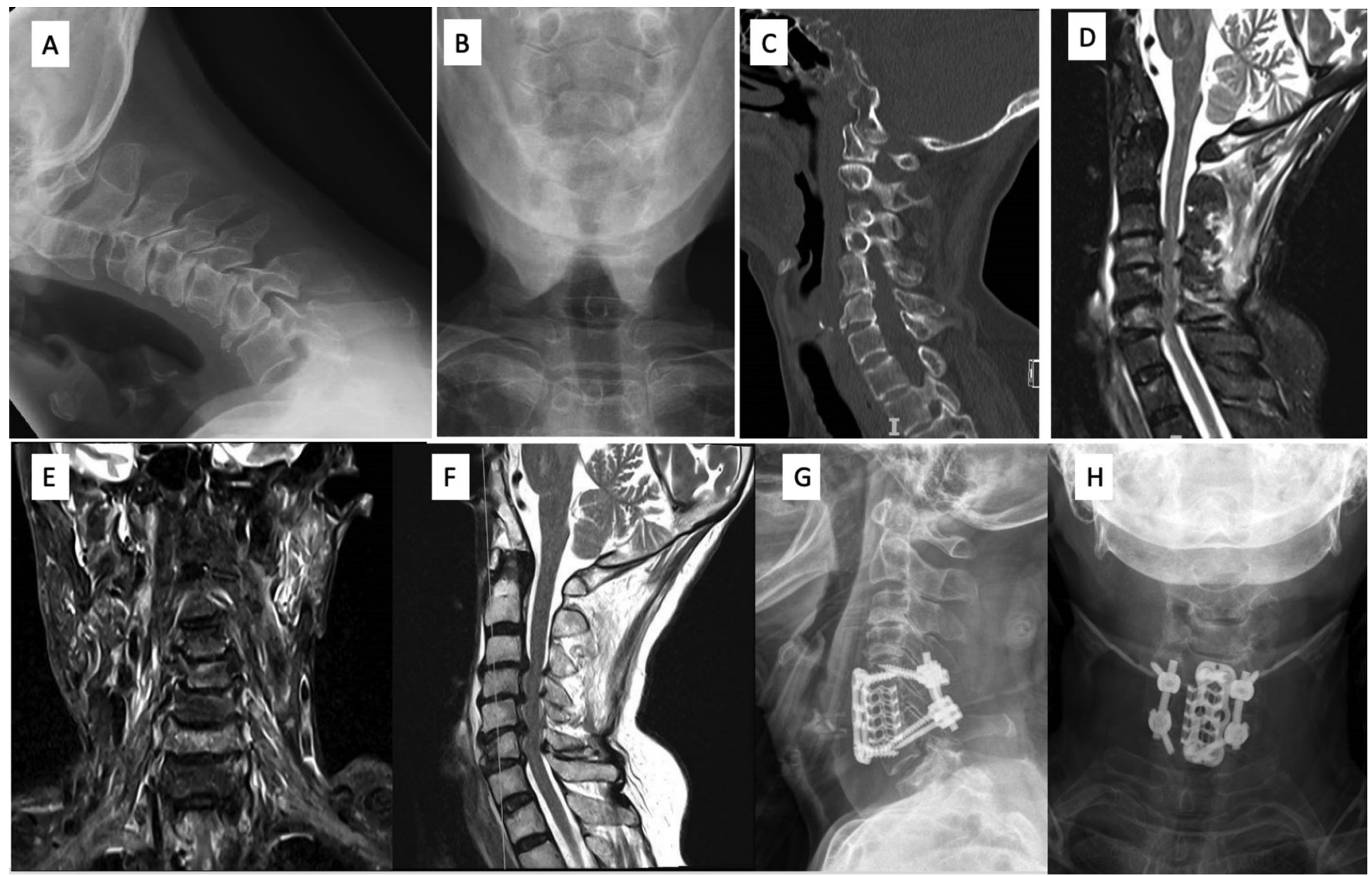

Fig. 1. A, B: pre-operative X-rays, C: sagittal CT views demonstrating the C6 flexion tear drop fracture. D: STIR image of the pre-operative MRI showing high signal intensity in the interspinous ligament between $\mathrm{C} 5$ and C6, where there is a transverse fracture in the body of the C6 spinous process, and high signal intensity in the C6 body. E: T1 weighted coronal view. F: T2 weighted sagittal view showing the corresponding MRI views demonstrating the C5-C6 interspinous ligament oedema and widening, as well as bony oedema on C6 vertebral body. G, H: post-operative radiographs demonstrating fracture fixation with restoration of cervical alignment. 
have been underestimated, as it will be difficult to keep the fracture $\mathrm{C} 6$ body reduced and maintain its height. The C6 spinous process split meant a loss of the posterior tension band system, and there was definite facet capsular disruption. The posterior distraction element makes this injury prone to further worsening kyphosis if the 3-column disruption was treated non-operatively.

The majority of cervical injuries reported after external spinal manipulation occurred in patients with ankylosing spondylitis. ${ }^{7,8}$ Ankylosis spondylitis causes a loss of elasticity in the spine due to syndesmophyte, which in turn causes an alteration in biomechanics with the spine becoming an osteoporotic long bone "bamboo" prone to fractures after trivial trauma. ${ }^{7,11}$ Ankylosing spondylitis patients are thus 3.5 times more likely to sustain spinal fractures than the unaffected population. ${ }^{8}$

Loss of bone mass in osteoporosis causes a decrease in mechanical strength of the bone and predisposes patients to fragility fractures. ${ }^{12}$ In the lumbar vertebrae, biomechanical studies have also demonstrated that the loss of vertebral height in patients with osteoporosis and degenerative disc disease lead to decreased stability of the spinal column with increased vertebral translation and rotation on spine mobilisation. ${ }^{10}$ Hence, we postulate that in our patient's case, osteoporosis was the main predisposing factor for fracture, with degenerative disco-vertebral changes as a possible contributing factor.

Apart from cervical fractures, neurological complications of external cervical manipulation has been described with an estimated incidence of up to 1 in $50,000 \cdot{ }^{13} \mathrm{~A}$ review by Turner et al. found 901 cases of vertebral artery dissection in patients following neck manipulation, out of which 707 patients subsequently developed stroke, with 26 reported deaths. ${ }^{3}$ Other reported causes of neurological damage post-manipulation included epidural haematoma ${ }^{2}$ and prolapsed intervertebral discs $^{4}$ causing cord compression. While most cases of manipulation and massage do not result in harm, the severe complications if they occur warrants caution by TCM practitioners.

No guidelines for external cervical manipulation have been implemented in Singapore thus far. There has been an increased worldwide recognition of the danger in injudicious practice. The French Society for Manual Orthopedic and Osteopathic Medicine (Société Française de Médecine Manuelle Orthopédique et Ostéopathique) published a consensus statement calling for careful 5 point pre-procedural assessment before manipulation and proper training to be completed by the alternative medicine practitioner. ${ }^{14}$ Osteoporosis and advanced age, found in our patient, were both listed as contraindications in the said statement. While it is beyond the scope of this report to discuss policy recommendations, stricter guidelines and increased regulation of alternative medicine practice can help to prevent injury.

In conclusion, Tui $\mathrm{Na}$ is a relatively safe traditional treatment option for relieving muscle pain and tightness. However, in patients with spinal pathologies such as osteoporosis and ankylosing spondylitis, forceful mechanical manipulation of the neck can cause catastrophic injuries to nerve, blood vessel and bone. Guidelines demarcating the limits and contraindications of Tui Na therapy have been adopted by TCM practitioners to prevent such injuries. It is important for all physicians to routinely enquire about usage of alternative medicine such as Tui $\mathrm{Na}$ by their patients and to offer appropriate advice on its benefits and risks. This is especially pertinent in our aging community as many elderly patients with concomitant osteoporosis seek alternative traditional treatment.

\section{REFERENCES}

1. Yang YJ, Zhang J, Hou Y, et al. Effectiveness and safety of Chinese massage therapy (Tui $\mathrm{Na}$ ) on post-stroke spasticity: a prospective multicenter randomized controlled trial. Clin Rehabil 2017;31:904-12.

2. Ryu JI, Han MH, Kim JM, et al. Cervical epidural hematoma that induced sudden paraparesis after cervical spine massage: Case report and literature review. World Neurosurg 2018;112:217-20.

3. Turner RC, Lucke-Wold BP, Boo S, et al. The potential dangers of neck manipulation \& risk for dissection and devastating stroke: an illustrative case \& review of the literature. Biomed Res Rev 2018;2:1-4.

4. Talluri SK, Talluri J, Besur S, et al. Catastrophic complication of chiropractic manipulation: a report of quadriparesis. Am J Med 2009; 122:e3-e4

5. Vaccaro AR, Koerner JD, Radcliff KE, et al. AO spine subaxial cervical spine injury classification system. Eur Spine J 2016;25:2173-84.

6. Patel AA, Dailey A, Brodke DS, et al. Subaxial cervical spine trauma classification: the subaxial injury classification system and case examples. Neurosurg Focus 2008;25:E8.

7. Abilash K, Mohd Q, Ahmad Z, et al. Fracture dislocation at C6-C7 level with quadriplegia after traditional massage in a patient with ankylosing spondylitis: a case report. Malays Orthop J 2017;11:75-7.

8. Liao CC, Chen LR. Anterior and posterior fixation of a cervical fracture induced by chiropractic spinal manipulation in ankylosing spondylitis: a case report. J Trauma 2007;63:E90-4.

9. Ea HK, Weber AJ, Yon F, et al. Osteoporotic fracture of the dens revealed by cervical manipulation. Joint Bone Spine 2004; $71: 246-50$ 
10. Guo Z, Chen W, Su Y, et al. Isolated unilateral vetebral pedicle fracture caused by a back massage in an elderly patient: a case report and literature review. Eur J Orthop Surg Traumatol 2013;23 Suppl 2:S149-53.

11. Hee HT, Thambiah J, Nather A, et al. A case report of neurologically unstable fracture of the lumbosacral spine in a patient with ankylosing spondylitis. Ann Acad Med Singap 2002;31:115-8.

12. Duan Y, Seeman E. Bone fragility in Asian and Caucasian men. Ann Acad Med Singap 2002;31:54-66.

13. Malone DG, Baldwin NG, Tomecek FJ, et al. Complications of cervical spine manipulation therapy: 5-year retrospective study in a single group practice. Neurosurg Focus 2002;13:ecp1.

14. Vautravers P, Maigne JY. Cervical spine manipulation and the precautionary principle. Joint Bone Spine 2000;67:272-6.
Glenys MN Poon, ${ }^{1}$, Keng Lin Wong, ${ }^{2,3}{ }^{M C I}$, FRCS, FAMS, Haobin $\underline{\text { Chen }},{ }^{2,3}{ }_{F R C S}$, Sandip Singh Saggi, ${ }^{2} M B B S$, Maksim Lai, ${ }^{2} M B B S$, Merng Koon Wong, ${ }^{2,3}{ }_{F R C S, ~ F A M S,}$ Daniel Chan, ${ }^{2}{ }_{F R C S}$

${ }^{1}$ Phase V Student, Yong Loo Lin School of Medicine, Singapore

${ }^{2}$ Department of Orthopaedic Surgery, Sengkang General Hospital, Singapore

${ }^{3}$ Musculoskeletal Sciences ACP, Singhealth-DukeNUS Academic Medical Centre, Singapore

Address for Correspondence: Asst Prof Keng Lin Wong, Department of Orthopaedic Surgery, Sengkang General Hospital, Sengkang General Hospital, 110 Sengkang East Way, Singapore 544886.

Email: francis.wong.k.1@singhealth.com.sg 